Revue

de Sémantique

et Pragmatique

\section{Revue de Sémantique et Pragmatique}

41-42 | 2017

Cadrage sur la variation, le changement lexical et le changement grammatical en français actuel

\title{
Alternance futur simple / futur périphrastique : variation et changement en français oral hexagonal
}

Lotfi Abouda et Marie Skrovec

\section{QpenEdition}

\section{Journals}

Édition électronique

URL : http://journals.openedition.org/rsp/479

DOI : $10.4000 /$ rsp. 479

ISSN : $2610-4377$

Éditeur

Presses universitaires d'Orléans

Édition imprimée

Date de publication : 1 décembre 2017

Pagination : 155-179

ISSN : $1285-4093$

Référence électronique

Lotfi Abouda et Marie Skrovec, «Alternance futur simple / futur périphrastique : variation et changement en français oral hexagonal », Revue de Sémantique et Pragmatique [En ligne], 41-42 | 2017, mis en ligne le 01 décembre 2018, consulté le 01 mai 2019. URL : http://journals.openedition.org/ rsp/479; DOI : 10.4000/rsp.479 


\section{Alternance futur SIMPLe / FUTUR PÉRIPHRASTIQUE : VARIATION ET CHANGEMENT EN FRANÇAIS ORAL HEXAGONAL}

Lotfi Abouda \& Marie Skrovec LLL (UMR 7270) - Université d'Orléans

INTRODUCTION

01, Emirkanian \& Sankoff 1986, Fleury \& Branca 2010, Jeanjean 1988, Laurendeau 2000, etc.),

itères sémantiques 
Lotfi Abouda \& Marie Skrovec

es entretiens, le profil des locuteurs interviewés en 
projetées (Abouda \& Baude 2007).

enre « entretiens » reste majoritaire, nous avons toutefois réussi à intégrer à hauteur de $20 \%$ deux autres genres interactionnels de « contrôle », sup 
avail d'annotation a exclusivement concerné l'identification des propriétés sémantiques des formes étudiées, que

ion des données. Plusieurs expériences d'auteurs antérieurs montrent aisément l'inadéquation d'une telle démarche simplifica 'identifier toutes les phrases de type

cun des énoncés pour éventuellement identifier la propriété en question («implication », dans notre terminologie, voir § 1.2.4.

pparaître dans des contextes syntaxiques diffé

ée entre FS et négation ne sera pas commentée ici, même si

\begin{tabular}{l|l|l|}
\hline & & \\
\hline & & \\
\hline & & \\
\hline
\end{tabular}


écise, un ensemble

émantique identifiée.

ple, "Previous accounts of the distribution of periphrastic and inflected fu

[...]." King \& Nadasti (2003 : 330).

s impending'

'objet 
Lotfi Abouda \& Marie Skrovec

re à midi ce soir quand je

gens très très riches qui vous

tendance à prendre un petit peu un style euh comme on croise souvent

d'une part, à spécifier les 
an 1988 , Confais $1990 \ldots$ ), alors que le FS servirait

nson mais dans des caves et donc avec force dégustation

ocès dans sa phase préparatoire) qui ne se rencontre qu'au FP, et ce qui est affirmé ici concernant le trait « lien avec le présent » qui peut se présenter aussi bien au FP qu' au FS. Si ces deux propriétés

vent déjà être réunies dans le présent, etc.), comme dans l'exemple (9) ci-dessous. 
Lotfi Abouda \& Marie Skrovec

e le procès au futur de t0, pour vérifier une hypo

leurs essentielles : le certain et le non certain, afin de vérifier l'une des hypo 
noncé au 
Lotfi Abouda \& Marie Skrovec

Dans l'emploi de type «modalisation du dire par un verbe de parole », que

ploi que nous proposons d'appeler "typicalisation" regroupe des 
mais vous parce que

dre attendu des choses » (Damourette \& Pichon,

nds le numéro de portable du chauffeur et il peut arriver n'im 
Lotfi Abouda \& Marie Skrovec

nelles » pourrait 
ttendu dans le cadre d'une hypothèse sur le changement : les plus jeunes uti

tion

en termes des CSP sur l'échelle adaptée parle peu (figure 3). On observe toujours

$s$ universitaires (deux 
Lotfi Abouda \& Marie Skrovec

$\mathrm{z}$ les jeunes pour la forme plus récente, mais éga

d'emploi

'entre eux, jugés particulièrement significatifs ou 
alors tenté de penser que la distribution, catégorique, reflète une complémenta-

t visible dans

résenter le

\begin{tabular}{|l|}
\hline $\begin{array}{l}\text { Proximité : répartition des valeurs en } \\
\text { pourcentage }\end{array}$ \\
lutôt proche plutôt éloigné \\
$\square F P \backsim F S$ \\
\hline FP
\end{tabular}

Aspect global : répartition des formes en poucentage

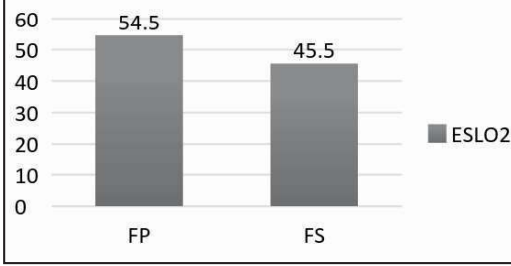


Lotfi Abouda \& Marie Skrovec

u FS et du

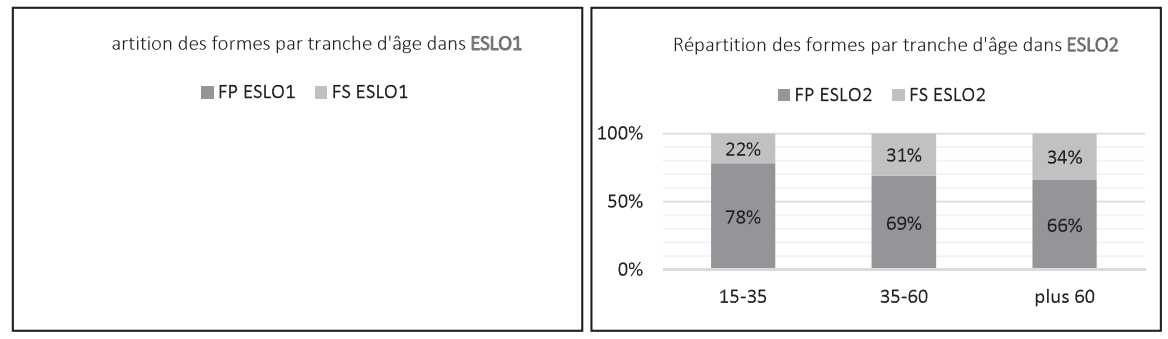




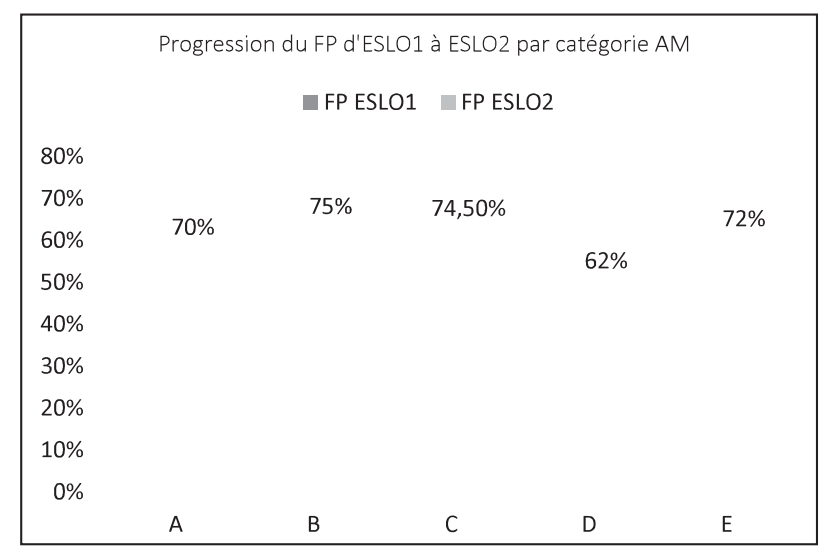

oyé que dans 
Lotfi Abouda \& Marie Skrovec

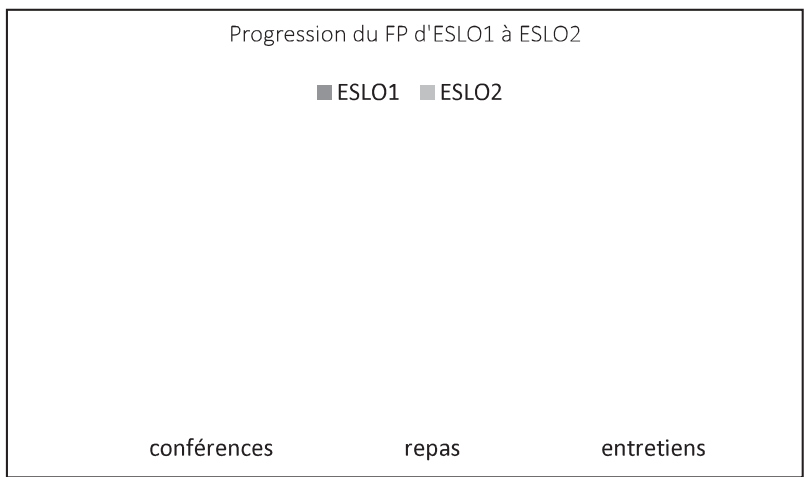

hybrides), les tendances brutes présentées ci-dessus (voir figure 7) montrent un FP qui progresse d'une façon importante

ter les tendances suivantes (figures 11 à 14) en indiquant 


partition par type
d'emploi
$\square f \backsim \mathrm{m}+\mathrm{fm}$

\begin{tabular}{|c|c|c|}
\hline & \multicolumn{2}{|c|}{$\begin{array}{l}\text { Progression du FS, répartition par type } \\
\text { d'emploi } \\
\text { f } \approx \mathrm{m}+\mathrm{fm}\end{array}$} \\
\hline 1200 & & \\
\hline 1000 & & \\
\hline 800 & \multirow[t]{2}{*}{453} & \\
\hline 600 & & \\
\hline 400 & \multirow[b]{2}{*}{526} & 89 \\
\hline 200 & & 372 \\
\hline & ESLO1 & ESLO2 \\
\hline
\end{tabular}

t pas de confirmer cette hypothèse puisque le FS, en 
Lotfi Abouda \& Marie Skrovec

ffaissement important au

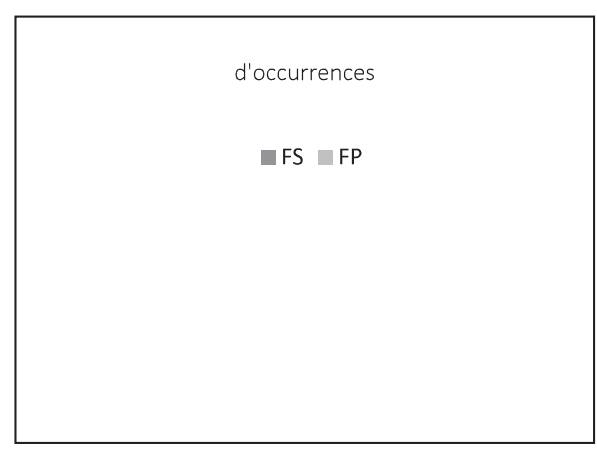

e en partie analogue au précédent, puisque,

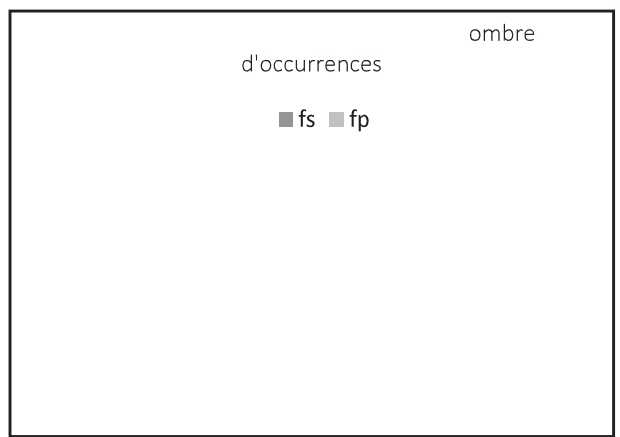


d'une perte de spécificité pour certains traits. En effet, l'une des valeurs possibles des trois attributs « Proximité », «Lien » et « Actualisation » est la valeur «non-pertinent », qui est attribuée à une occurrence chaque fois qu'aucune valeur spécifique ne peut lui être attribuée vis-à-vis du trait envisagé. Or, si on envisage ici les deux traits «Lien avec le Présent » et « Proximité avec T 
Lotfi Abouda \& Marie Skrovec

cificité du FP, nous parait d'au-

st assumée que par des FS : 
ribution. Nous avons ainsi

ux types d'imparfait atténuatif »,

, in F. Rastier et M. Ballabriga (dir.), Corpus en

synthétique et analytique du futur. Étude de la variable modale dans un corpus oral micro-diachronique », 
ngage et Ana

tuelles »,

ce normative ? », Actes du Colloque international d études romanes «Normes et grammaticalisation : le cas des langues romanes», Sofia, 20-21 novembre 2015.

, « Retour sur le futur dans les corpus de français parlé »,

français contemporain, de ses cendres médiévales ? », C. Guillot et

fet de sens extraordinaire par aller et venir »,

lé » in

a, S. (2010), «Une expérience de collaboration entre linguiste et 
tform:Building Open-Source Textual Analysis Soft

tur simple et le futur périphrastique en français parlé : Étude distributionnelle » in C. Blanche-Benveniste et al.

he future in Acadian French",

Holtus G., Metzeltin M., Schmitt Ch. (éds),

hèse

modale »,

f classifying the population

al reference in French". 